\title{
EFFECT OF CARBIMAZOLE ON THE STRUCTURE OF THE OVARY OF THE ADULT ALBINO RAT
}

\author{
By \\ Mostafa Mohammed Refat Hussein Mahran**, Moheb Farid Mounir \\ Rafla*, Hassan Mostafa Serry*, Ahmed Yehia Mostafa Awad* and Inas \\ Abd El-Hafiz* \\ **Department of Anatomy and Embryology, Faculty of Medicine, Misr University for \\ Science \& Technology \\ *Department of Anatomy and Embryology, Faculty of Medicine, Ain-Shams University \\ Corresponding author: Mostafa Mohammed Refat Hussein Mahran
}

Mobile: +02 01003616519, E-mail: mostafamhtc@gmail.com

\begin{abstract}
Background: Thyroid hormones are key regulators for a variety of biological processes including reproduction. The alterations in thyroid hormones levels were found to disturb the proper functioning of the ovary impairing fertility.
\end{abstract}

Objective: To investigate the effect of Carbimazole induced hypothyroidism on the ovaries of adult female albino rats.

Materials and methods: Forty five adult female albino rats were used and were classified into three equal groups: Group I (Control group):received $0.5 \mathrm{ml}$ of distilled water every day orally by gastric tube for three months, Group II (Hypothyroid group) received Carbimazole at a dose of 5mg dissolved in distilled water every day orally by gastric tube for three months to induce hypothyroidism, and Group III (Recovery Group) ceased to receive Carbimazole for three months to examine the changes after the stoppage of the treatment.

The changes were tested by measuring the serum levels of TSH, T4 and T3, FSH, LH, E2 and PRL. Moreover, the effects on the structure of the ovary were investigated via histological studies by using Hematoxylin and Eosin, and Masson's Trichrome along with immuno-histochemical studies using Vimentin and $\alpha$ - smooth muscle actin.

Results: The examination of ovarian specimens revealed a change in the normal architecture of the ovary, where the ovarian cortex showed an apparent decrease in the number of growing follicles with large fluidfilled cystic follicles and multiple atretic follicles. Marked condensation of the interstitial cells among the follicular component of the cortex was identified with engorged blood vessels which were reversed upon stoppage of the intake of the drug.

Conclusion: The use of Carbimazole in induction of hypothyroidism affected the normal functioning of the ovary via acting on different levels.

Keywords: Carbimazole, Ovary, Hypothyroidism, Vimentin, Alpha smooth muscle actin.

\section{INTRODUCTION}

Carbimazole, an anti-thyroid agent, was selected to induce a state of hypothyroidism in adult female albino rats through its effect on reducing the thyroid hormone synthesis (Mckay et al., 2010). It should be noted that since hypothyroidism is uncommon to occur naturally in rats so its effects could only be assessed by 
experimentally inducing hypothyroidism (Patel et al., 2013). Thyroid hormones were found to possess a strong impact on sexual and reproductive functions in both humans and animals (Nelson et al., 2011).

The ovary is a dynamic organ in both rats and human beings. Furthermore, the authors added that the ovary is very responsive to hormonal influences that can improve or alter its functions throughout the estrus cycle in rodents or menstrual and ovarian cycles in humans (Treuting et al., 2018) Moreover, thyroid disorders can be presented as hyperthyroidism or more commonly as hypothyroidism. Both hyperthyroidism and hypothyroidism were found to be associated with numerous morphological and physiological disorders (Silva et al., 2018).

Hypothyroidism in females was found to impair fertility by direct and indirect effects on the endometrium and ovaries through various reproductive disorders (Mcdermott, 2012). In addition, thyroid dysfunction was found to be associated with subfertility both in men and women (Dittrich et al., 2011).

The present work aimed to investigate the effect of Carbimazole induced hypothyroidism on the ovaries of adult female albino rats.

\section{MATERIALS AND METHODS}

The plan of the study was done according to guidelines of the Committee of Animal Research Ethics (CARE) of the Faculty of Medicine at Ain-Shams University.

Forty five adult female albino SpragueDawley rats, each weighing 180-220 grams, were used for the current study, and they were kept isolated from males one month before starting the experiment and all the female albino rats were kept isolated from each other in separate cages throughout the experiment.

The animals were housed in suitable environment having free access to food (rat chaw) and water and were all exposed to normal light-dark cycle, good ventilation and temperature of 22o-25o C.

Vaginal smears were taken from the rats and stained with methylene blue to ensure the regularity of the estrus cycle. Those who showed three successive cycles (4 days for each cycle) were the ones of choice for the experiment and rats showing irregular cycles were excluded.

The body weight of each rat was measured at the beginning and at the end of the experiment.

The experimental rats were randomly divided into 3 equal groups:

\section{Group I (Euthyroid, Control group)} received $0.5 \mathrm{ml}$ of distilled water every day orally by gastric tube for three months.

2. Group II (Hypothyroid group) received Carbimazole at a dose of $5 \mathrm{mg}$ dissolved in distilled water every day orally by gastric tube for three months to induce hypothyroidism (Zaidi et al., 2004).

3. Group III (Recovery Group) ceased to receive carbimazole for three months to show the changes after stopping the drug.

Throughout the experiment, a weekly blood sample was collected through a retroorbital vein in a plain tube, left for 
ten minutes, and then centrifuged. Serum was used to determine the levels of thyroid stimulating hormone (TSH), thyroxine (T4) and tri-iodothyronine (T3) by ELISA (Enzyme Linked Immunosorbent Assay) to prove the induction of the hypothyroid state. Moreover, follicular stimulating hormone (FSH), Luteinizing Hormone (LH), estrogen (E2) and prolactin (PRL) were also measured.

The ovarian specimens were separated and washed by saline then placed in Bouin solution for fixation for twenty four hours and then processed for preparing paraffin blocks using conventional techniques for histological studies. Serial sections within the tissue block were obtained using the rotatory microtome and section thickness was between 5-6 $\mu \mathrm{m}$.

The sections were examined by Olympus light microscope and photographed in the Anatomy Department of the Faculty of Medicine, Ain-Shams University starting December 2015 till December 2020.

Sections were processed for histological staining by using hematoxylin and Eosin (Hx\&E) and Masson's Trichrome (MT).

The staining protocol using vimentin was done by using four micron paraffinembedded tissue sections which were applied to glass slides coated with $0.1 \%$ poly-D-lysine. The tissue sections were immuno-stained using an avidin-biotin peroxidase complex (ABC) method.

The primary antibodies were rabbit anti-vimentin (Monoclonal Mouse AntiVimentin, Clone V9; DAKO, Denmark) at 1:250 dilution. The negative control was
Dako Mouse IgG1, code number X 0931, diluted to the same mouse $\mathrm{IgG}$ concentration as the primary antibody.

The sections were rehydrated by sequential immersion in xylene, graded concentrations of ethanol and tap water. Slides were kept in citrate buffer $(\mathrm{pH} 9)$ and two cycles of heat retrieval were done in oven at $99^{\circ} \mathrm{C}$ for ten and five minutes, respectively.

Furthermore, the slides were washed in Tris buffer ( $\mathrm{pH} 7.8)$. All tissue sections were incubated with hydrogen peroxide for 10 minutes to eliminate endogenous peroxidase activity. Sections were washed thrice in Tris buffer, followed by 30 minutes incubation with vimentin antibodies. Moreover, the secondary antibody (Dako REALTM EnvisionTM) was added after washings with Tris buffer for 40 minutes.

At the end, Diaminobenzidine (DAB) was added for 10 minutes, followed by counterstaining with hematoxylin for two minutes, sequential immersions in xylene and alcohol and mounting with distyrene plastisizer xylene (DPX) (Salvetti et al. 2004).

The steps of immuno-staining using alpha smooth muscle actin began by antigen retrieval using $0.125 \%$ trypsin solution at RT for $15 \mathrm{~min}$, followed by blocking with 5\% normal horse serum (for $\alpha-S M$ actin) and incubation with primary antibodies overnight at $48^{\circ} \mathrm{C}$.

The primary antibodies used to label different actin isoforms were: mouse antisera against a-SM actin (1:50, Clone 1A4; Dako Diagnostics). For the negative controls, Chrom-Pure nonspecific mouse or sheep IgGs (Jackson Immuno-Research 
Laboratories, Inc., West Grove, PA) were used at the same concentration, and sections were also incubated with secondary antibodies in the absence of primary antibodies.

Moreover, the secondary antibodies used for detection were biotinylated antimouse (1:200; Vector, Burlingame, CA) and donkey anti-sheep-HRP-conjugated (1:1000; Serotec, Oxford, UK). Final visualization was achieved using Vectastain Elite Kit (Vector, Burlingame, CA).

Finally, counterstaining with Harris' Hematoxylin (Sigma Di-agnostics, St. Louis, MO) was carried out before slides were mounted with Cytoseal XYL (Ricard-Allan Scientific, Kalamazoo, MI) (Hirschberg et al., 2012).

Based on the intensity of color produced by immuno-staining in more than $50 \%$ of cell population using the three stains, the color grading was scored as follows: $1+$ weak staining, $2+$ moderate staining and $3+$ strong (intense) staining.

\section{Statistical analysis:}

Statistical analysis for the laboratory results was done by using Statistical Package for the Social Sciences Software - version 13.0 (SPSS), data are presented as mean \pm standard deviation (SD), median (IQR), Kruskal wallis test, Post hoc analysis by LSD and one-way analysis of variance (ANOVA) were employed to compare the different groups with each other.

The significance of the data by the probability (P. value) was non-significant when $\mathrm{P}>0.05$, significant when $\mathrm{P} \leq 0.05$ and was considered highly significant when $P \leq 0.001$. Data were represented in tables and histograms, prepared by using MS Excel 2013 (Gao, 2020). 


\section{RESULTS}

I. Control group: Light microscopic examination of the adult female albino rat showed the ovarian parenchyma divided into a peripheral cortex surrounding a central medulla with no sharp demarcation between them. The ovarian cortex was covered by surface epithelium and underneath it a layer called the tunica albuginea. In addition, below the tunica albuginea, multiple ovarian follicles of various stages of development were identified in the stroma of the cortex like Primordial follicles, Primary follicles (early and late), secondary follicles, and mature Graafian follicle. Follicles that weren't clearly identified were referred to as growing follicles with numerous blood vessels present at the medulla (Figures 1 and 2).

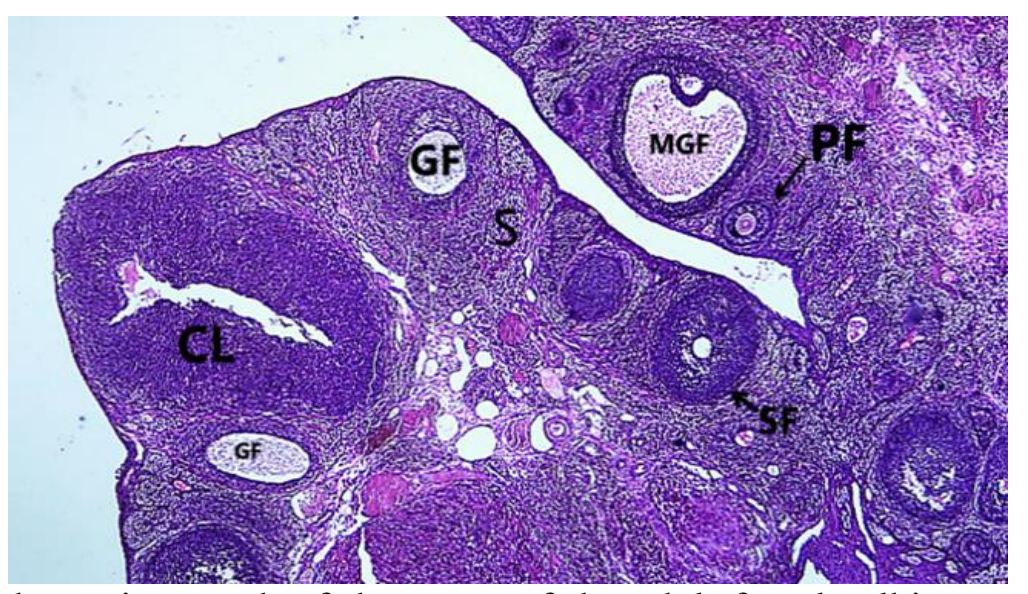

Figure (1): A photomicrograph of the ovary of the adult female albino rat of the control group showing ovarian follicles of various sizes distributed in the stroma (S) of the cortex. The follicles are primary follicle (PF), Secondary follicle (SF), growing follicle (GF), Mature Graafian follicle (MGF), and corpus luteum (CL) (Hx\&E, 40X).

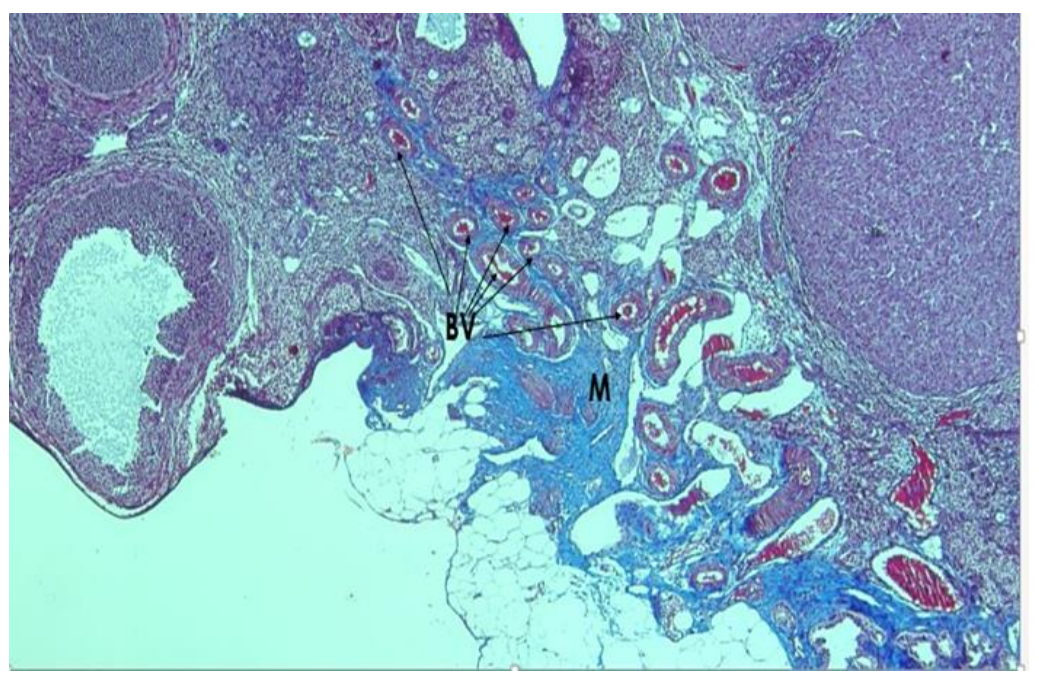

Figure (2): A photomicrograph of the ovary of adult female albino rat of the control group showing the medulla $(\mathrm{M})$ which stained blue due to presence of collagen fibers and the medulla is characterized by the presence of numerous blood vessels (BV) (M.T, 40X). 
II. Experimental groups:

a. Group II: Examination of the ovarian sections showed that the ovary kept the general architecture of being divided into a peripheral cortex surrounding a central medulla. However, the peripheral cortex was invaded by numerous large sized growing follicles and corpora lutea squeezing the stroma and medulla with marked condensation of the interstitial cells along with marked congestion of cortical and medullary blood vessels (Figures 3 and 4).

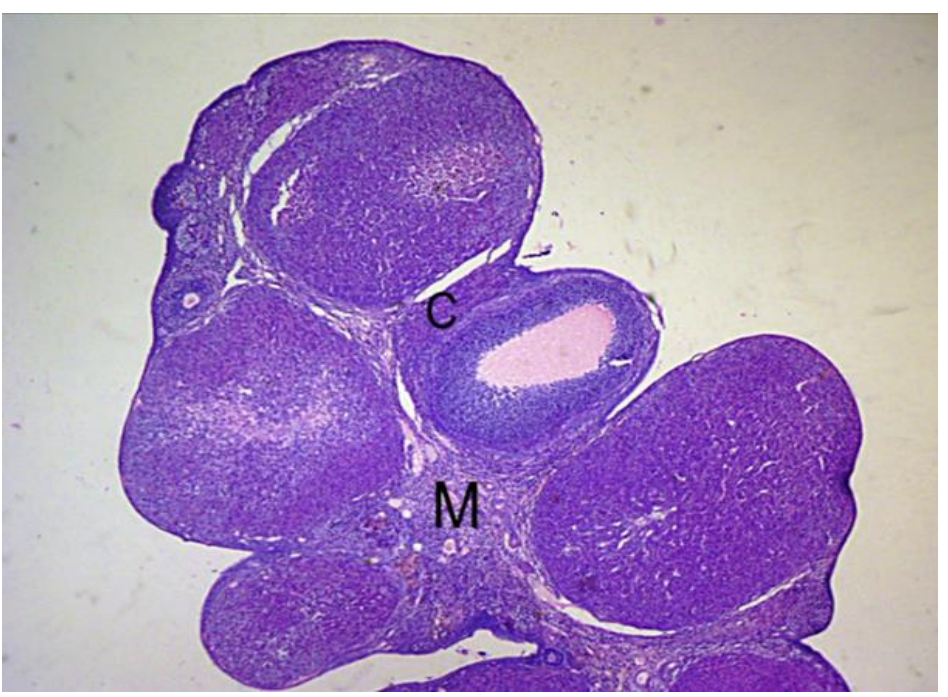

Figure (3): A photomicrograph of the ovary of adult female albino rat of the experimental Group II showing that the ovary kept the general architecture of being divided into a peripheral cortex (C) surrounding a central medulla (M) (Hx\&E, 40X).

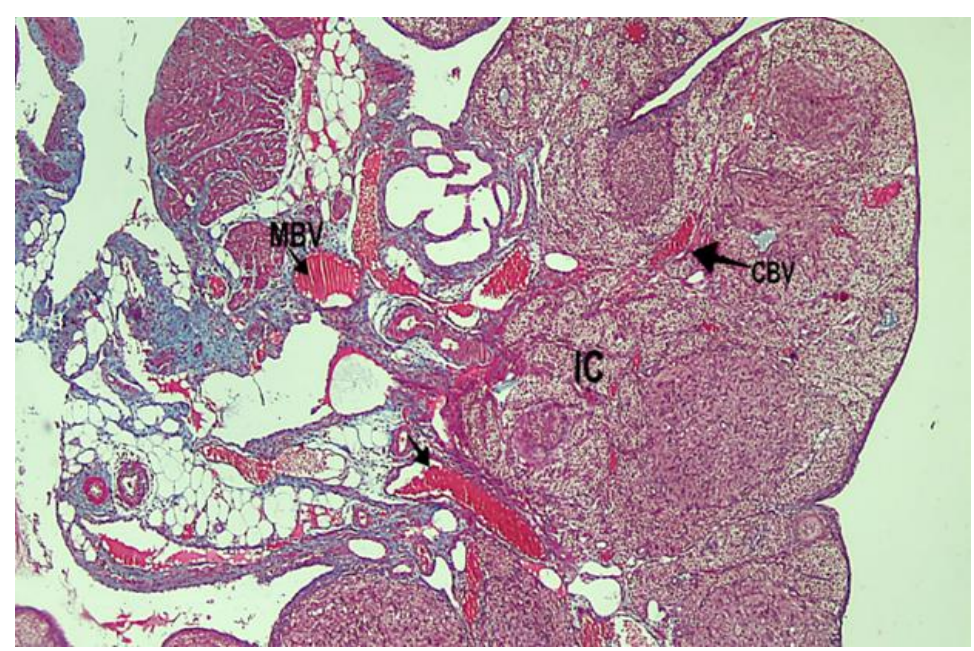

Figure (4): A photomicrograph of the ovary of adult female albino rat experimental Group II showing marked condensation of the interstitial cells (IC) among the follicular component of the cortex. Note the marked congestion of cortical (CBV) and medullary blood vessels (MBV) (M.T,40X). 
b. Group III: Large fluid-filled follicles were spotted amongst the follicles that are various stages of development squeezing the stroma

between them and the follicles (Figure 5) with engorged medullary blood vessels (Figure 6).

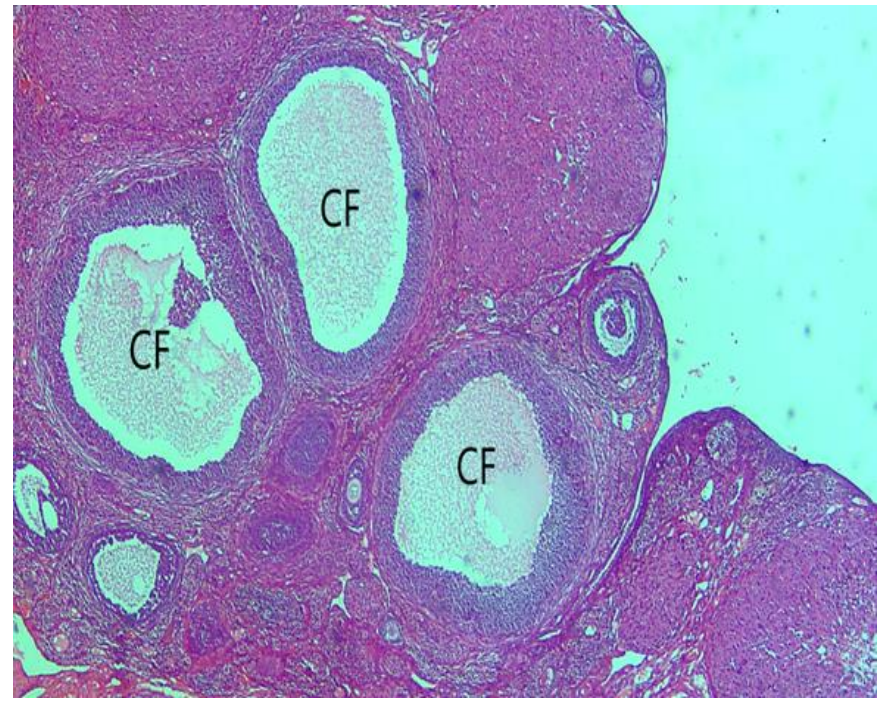

Figure (5): A photomicrograph of the ovary of the adult female albino rat of the experimental Group III showing large fluid filled cystic follicles (CF) (H\&E, 40X).

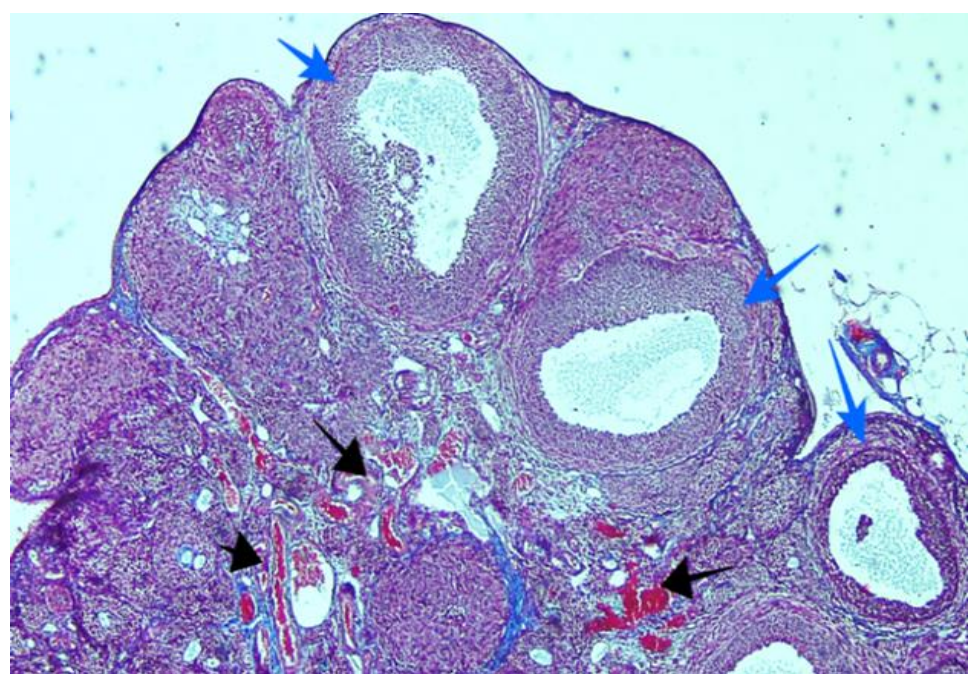

Figure (6): A photomicrograph of the ovary of adult female albino rat of the experimental Group III showing multiple cystic follicles (blue arrows) within the cortex with engorged medullary blood vessels (black arrows) (M.T, 40X). 


\section{MOSTAFA M. MAHRAN et al.,}

\section{HISTOCHEMICAL RESULTS}

I. Control group

1. Vimentin: The granulosa of healthy growing follicles of various stages of development were negatively stained along with their follicular fluid and the surrounding stroma. In addition, the interstitial cells showed moderate positive staining with dense staining around atretic follicles, blood vessels and corpora lutea. In addition, the atretic follicle showed positive staining of its granulosa along with its follicular fluid (Figure 7).

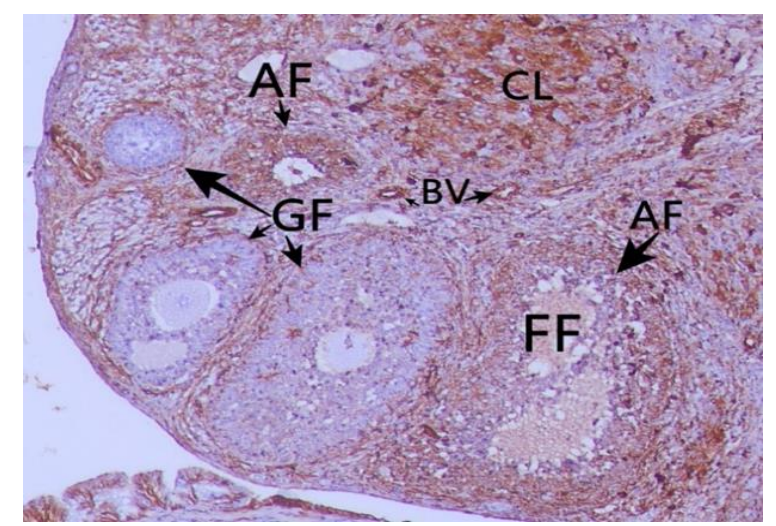

Figure (7): A photomicrograph of the ovary of the control group showing, growing follicles (GF) of various stages with dense staining around an atretic follicle (AF) positive staining of follicular fluid (FF) which is not the situation with a growing follicle where the follicular fluid is not stained in healthy growing follicle and moderate staining of stromal cells with strong staining around blood vessels (BV) and notice positive staining of the corpus luteum (CL) (Vimentin, 100X).

2. Alpha smooth muscle actin: addition, the granulosa of the secondary Examination of the ovaries belonging to follicle showed mild positive staining, the control group revealed that the surface along with moderate positive staining of epithelium, tunica albuginea and the granulosa of larger growing follicle, interstitial cells were negatively stained the follicular fluid as well as the corpus along with intense positive staining of the luteum (Figure 8). stroma surrounding the follicles. In

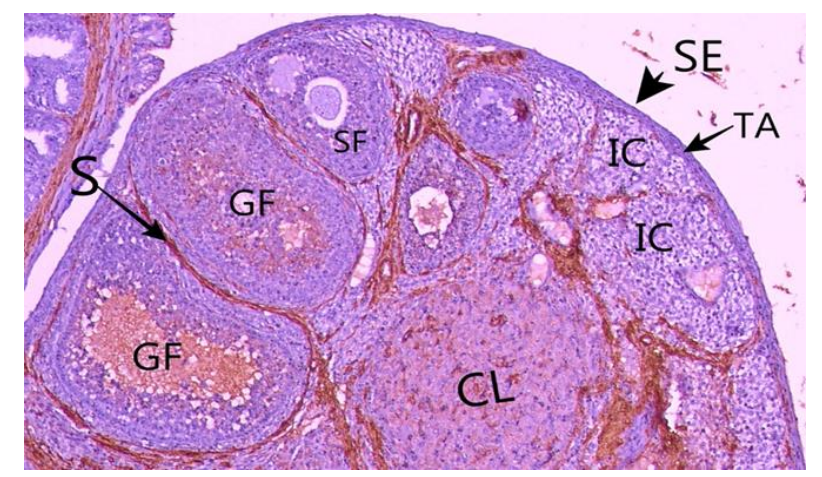

Figure (8): A photomicrograph of the ovary of the control group showing negative staining of the surface epithelium (SE), tunica albuginea (TA) and interstitial cells (IC). Notice the intense positive staining of the stroma $(S)$ and mild positive staining of granulosa of the secondary follicle (SF) with moderate staining of the granulosa of the growing follicles (GF) and the follicular fluid along with the corpus luteum (CL) ( $\alpha$ SMA, X100). 
II. Experimental groups:

a. Group II:

1. Vimentin: The corpus luteum shows moderate positive staining along with positive staining of the interstitial cells and around blood vessels, with negative staining for growing follicle and stroma. It should be noted that the staining pattern was the same as in the control group but lesser in intensity (Figure 9).

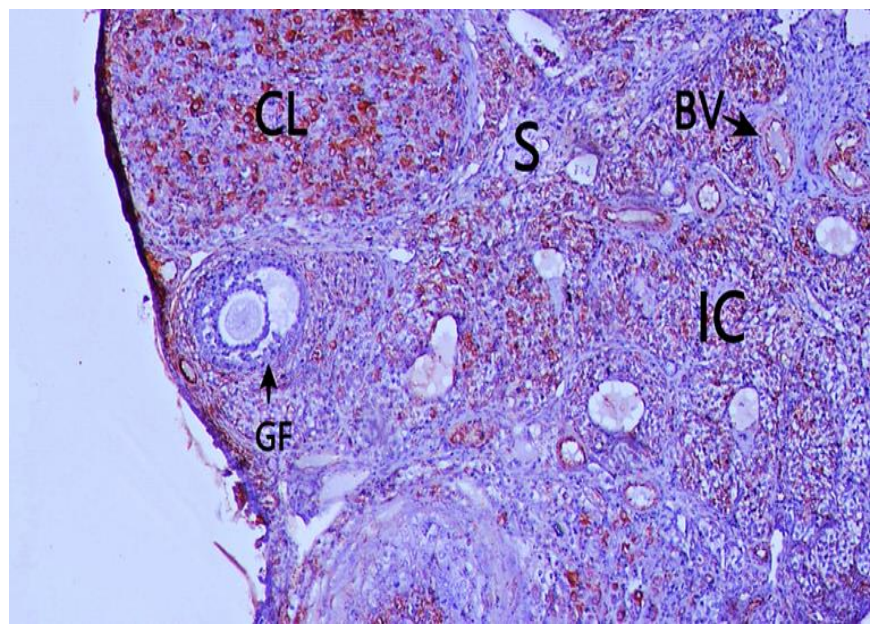

Figure (9): A photomicrograph of the ovary of the adult female albino rat of the experimental group II, where the corpus luteum (CL) shows moderate staining, and positive staining of the interstitial cells (IC) and around blood vessels (BV), with negative staining for growing follicle (GF) and stroma (S) (Vimentin, 100X).

2. Alpha smooth muscle actin: Examination of ovarian specimens showed positive staining around cortical blood vessels similar in pattern to that of the control group. Additionally, the corpora lutea showed focal to mild positive staining with negative staining of the primary follicle and the interstitial cells (Figure 10).

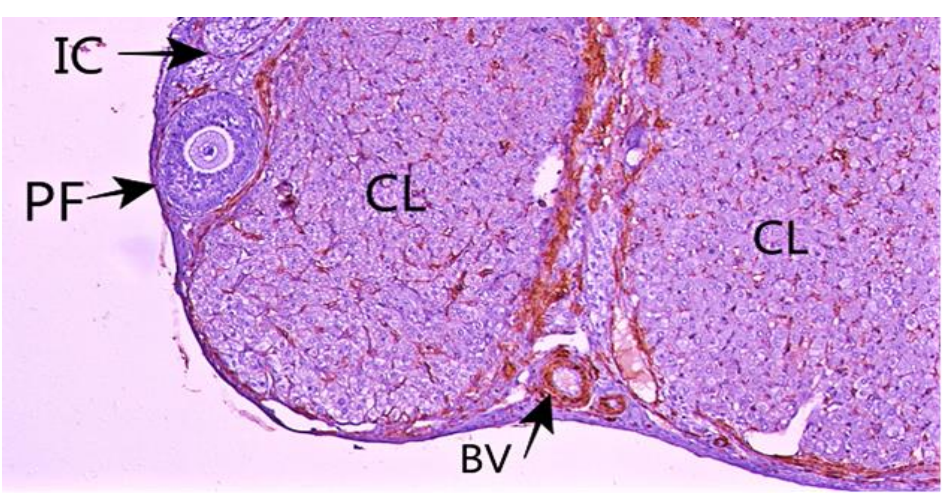

Figure (10): A photomicrograph of the ovary of the adult female albino rat of the experimental group II showing focal to mild positive staining of the corpora lutea(CL) and strong positive staining around blood vessels(BV) and negative staining of primary follicle (PF) and interstitial cells (IC) ( $\boldsymbol{\alpha S M A , ~ X 1 0 0 ) . ~}$ 
2. Group III:

1. Vimentin: The corpus luteum showed intense staining of the granulosa cells and around blood vessels within the corpus luteum with mild to moderate staining around atretic follicles and negative staining of the surrounding stroma (Figure 11).

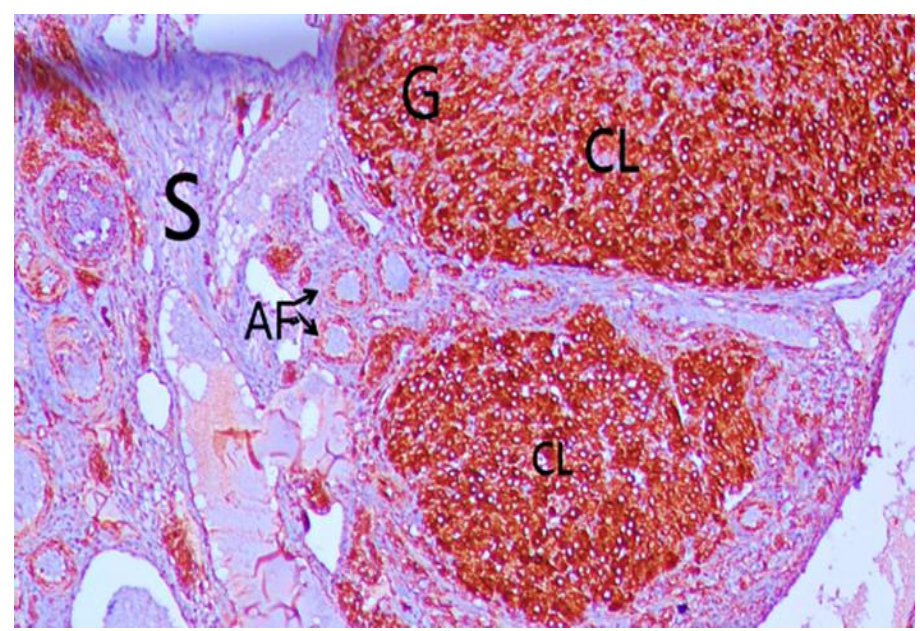

Figure (11): A photomicrograph of the ovary of the adult female albino rat of the experimental group III showing dense staining of granulosa $(\mathrm{G})$, of corpus luteum (CL), negative staining of the stroma $(S)$ with mild staining around atretic follicles (AF) (vimentin, 100X).

2. Alpha smooth muscle actin: The granulosa of the growing follicle showed mild positive staining which increased in pattern towards the follicular cavity accompanied by negative staining of the primary follicle and the atretic follicle. It was also noted that the granulosa of the corpus luteum showed patchy mild to negative staining pattern (Figure 12).

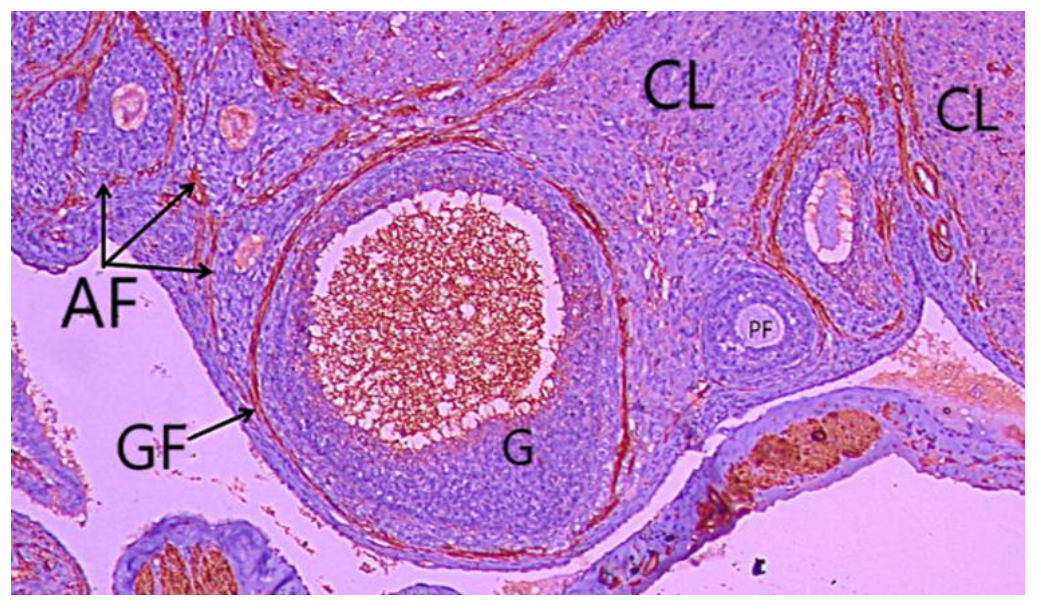

Figure (12): A photomicrograph of the ovary of the adult female albino rat of the experimental group III showing negative staining of the atretic follicles (AF), primary follicle (PF) and patchy mild positive staining of the granulosa $(G)$ of a growing follicle (GF) with patchy mild to negative staining of corpora lutea (CL) ( $\alpha$ SMA, 100X). 


\section{STATISTICAL RESULTS}

1. Estimation of TSH, TT3 and TT4 levels: Comparison of the means of the TSH level among the three groups of the study revealed significant statistical increase in group II compared with the control group. Group III showed significant statistical increase in means of TSH level compared with the control group, but significant statistical decrease compared with group II.

Regarding TT3, comparison revealed significant statistical decrease in group II compared with the control group. Group III showed significant statistical increase in means of TT3 level compared with the control group and group II.

As for TT4, comparison revealed significant statistical decrease in group II compared with the control group. Group III showed significant statistical decrease in means of TT4 level compared with the control group and group II (Table 1 and 2).

Table (1): Comparison between control group, group II and group III regarding TSH, TT3 and TT4

\begin{tabular}{|c|c|c|c|c|c|}
\hline \multicolumn{2}{|c|}{ Groups } & Control group & Group II & Group III & P-value \\
\hline $\begin{array}{c}\text { Parameters } \\
\text { TSH } \\
(\mathrm{IU} / \mathrm{Ml})\end{array}$ & Mean \pm SD & $2.34 \pm 1.02$ & $10.87 \pm 4.19$ & $2.63 \pm 1.02$ & 0.00 \\
\hline TT3 (ng/ml) & $\begin{array}{c}\text { Median } \\
(\mathrm{IQR})\end{array}$ & $1.84(1.12-2.13)$ & $0.31(0.29-0.4)$ & $2.89(2.43-3.1)$ & 0.00 \\
\hline TT4 (ug/dl) & $\begin{array}{c}\text { Median } \\
(\mathrm{IQR})\end{array}$ & $9.52(8.9-10.16)$ & $3.26(2.21-4.44)$ & $0.92(0.84-1.01)$ & 0.00 \\
\hline
\end{tabular}

P-value >0.05: Non significant; P-value <0.05: Significant; P-value< 0.01: highly significant

Table (2): Post hoc analysis comparing between control group, group II and group III regarding TSH, TT3 and TT4

\begin{tabular}{|c|c|c|c|}
\hline Post Hoc analysis & P1 & P2 & P3 \\
\hline TSH (IU/MI) & $\mathbf{0 . 0 0 0}$ & $\mathbf{0 . 8 9 0}$ & $\mathbf{0 . 0 0 0}$ \\
\hline TT3 (ng/ml) & $\mathbf{0 . 0 0 0}$ & $\mathbf{0 . 0 1 6}$ & $\mathbf{0 . 0 0 1}$ \\
\hline TT4 (ug/dl) & $\mathbf{0 . 0 0 0}$ & $\mathbf{0 . 0 0 5}$ & $\mathbf{0 . 0 0 2}$ \\
\hline
\end{tabular}

P1: Control Vs Group II, P2: Control group Vs Group III, P3: Group II Vs Group III

2. Estimation of FSH and LH levels: Comparison of the means of the FSH level among the three groups of the study revealed significant statistical decrease in group II compared with the control group. Group III showed significant statistical decrease in means of FSH level compared with the control group, but significant statistical increase compared with group II.
Regarding LH, comparison revealed significant statistical increase in group II compared with the control group. Group III showed significant statistical increase in means of LH level compared with the control group but significant statistical decrease compared with group II (Table $\mathbf{3}$ and 4). 
MOSTAFA M. MAHRAN et al.,

Table (3): Comparison between control group, group II and group III regarding FSH and LH

\begin{tabular}{|c|c|c|c|c|c|}
\hline \multicolumn{2}{|l|}{ Groups } & Control group & Group II & Group III & P-value \\
\hline FSH $(\mathrm{mIu} / \mathrm{ml})$ & Median (IQR) & $5.86(5.12-7.12)$ & $1.44(1.01-2.01)$ & $4.24(2.84-6.26)$ & 0.000 \\
\hline LH $(\mathrm{mIu} / \mathrm{ml})$ & Mean \pm SD & $7.08 \pm 2.10$ & $12.56 \pm 5.91$ & $9.02 \pm 2.75$ & 0.015 \\
\hline
\end{tabular}

P-value >0.05: Non significant; P-value <0.05 Significant; P-value $<0.01$ : highly significant

Table (4): Post hoc analysis comparing between control group, group II and group III regarding FSH and LH

\begin{tabular}{|r|c|c|c|}
\hline $\begin{array}{r}\text { Post Hoc analysis } \\
\text { By LSD }\end{array}$ & P1 & P2 & P3 \\
\hline Parameter & & & \\
\hline FSH $(\mathrm{mIu} / \mathrm{ml})$ & 0.000 & 0.203 & 0.001 \\
\hline $\mathrm{LH}(\mathrm{mIu} / \mathrm{ml})$ & 0.006 & 0.525 & 0.202 \\
\hline
\end{tabular}

3. Estimation of Prolactin (PRL) and Estrogen (E2) levels: Comparison of the means of the PRL level among the three groups of the study revealed significant statistical increase in group II compared with the control group. Group III showed significant statistical decrease in means of PRL level compared with the control group as well as group II.
Regarding E2, comparison revealed significant statistical decrease in group II compared with the control group. Group III showed significant statistical decrease in means of E2 level compared with the control group but significant statistical increase compared with group II (Table 5 and 6 ).

Table (5): Comparison between control group, group II and group III regarding PRL and E2

\begin{tabular}{|c|c|c|c|c|c|}
\hline \multicolumn{2}{|c|}{ Groups } & Control group & Group II & Group III & P-value \\
\hline $\begin{array}{c}\text { PRL } \\
(\mathrm{ng} / \mathrm{ml})\end{array}$ & Mean $\pm \mathrm{SD}$ & $13.52 \pm 5.13$ & $21.65 \pm 9.53$ & $10.63 \pm 4.27$ & 0.007 \\
\hline E2 $(\mathrm{pg} / \mathrm{ml})$ & $\begin{array}{c}\text { Median } \\
(\mathrm{IQR})\end{array}$ & $85.1(74-100.3)$ & $12.6(10-16.8)$ & $32.1(30.1-69.8)$ & 0.000 \\
\hline
\end{tabular}

P-value >0.05: Non significant; P-value <0.05: Significant; P-value < 0.01: highly significant

Table (6): Post hoc analysis comparing between control group, group II and group III regarding PRL and E2

\begin{tabular}{|c|c|c|c|}
\hline $\begin{array}{r}\text { Post Hoc analysis } \\
\text { By LSD }\end{array}$ & P1 & P2 & P3 \\
\hline Parameter & 0.012 & 0.566 & 0.019 \\
\hline PRL (ng/ml) & 0.000 & 0.090 & 0.002 \\
\hline
\end{tabular}




\section{DISCUSSION}

Thyroid hormones are the key regulators for a variety of biological processes, including growth, metabolism, development and differentiation in various tissues and organs including the ovary (Brent, 2012).

The present study aimed at investigating effect of the oral intake of Carbimazole for three months using gastric tube on the structure of the ovary of the adult female albino rat. Carbimazole, an anti-thyroid drug which is metabolized into Methimazole that blocks the thyroid hormogenesis and leads to experimentally induced hypothyroidism. The occurrence of hypothyroidism was confirmed by lab results that showed a decrease in the levels of T3 and T4 with an increase in TSH levels in accordance with the report of Treesh and Khair (2014). Furthermore, we investigated other hormones, such as Prolactin, LH, FSH and Estrogen E2, to gain a wider image about the changes which would happen and can affect the structure of the ovary. The present work recorded a decrease in FSH levels between the control group and group II that explained the decrease in number of follicles. Increased levels of LH were noticed in group II which explained the presence of multiple corpora lutea confirming the findings of Silva et al. (2018).

In addition, prolactin levels increased in group II more than that of the control group confirming the occurrence of hyperprolactinemia that created a state of pseudo-pregnancy which might be attributable to the changes that occurred which was also found by the work of
Canipari et al. (2019). Meanwhile, estrogen levels decreased in group II which was held responsible for the increased number of atretic follicles and the decrease in number of growing follicles since estrogen plays an important role in viability of follicular cells and their resistance to apoptosis.

The differences in lab results between group II and control groups showed the effect of hypothyroidism on other hormones and how hypothyroidism disturbed the normal functioning of pituitary-ovarian axis that affected follicular maturation and led to cystic formations which is in accordance with the work of Ajayi et al. (2013). In addition, these changes were found reversible upon the stoppage of Carbimazole intake for three months.

Our present work noticed a moderate increase in weight in rats that underwent treatment with Carbimazole which was about 5 to 10 grams increase in weight from the beginning of the experiment till sacrifice in comparison with the control rats, this agreed with the work of Nida et al. (2016).

The ovarian general architecture being divided into a peripheral cortex surrounding a central medulla was preserved in the three groups. However, the cortex showed an apparent decrease in the number of growing follicles in group II compared to control group and group III. The changes in the numbers of growing follicles were attributed to the disturbed estrus cycle along with hormonal disturbances which was also found by Hapon et al. (2010).

In addition, multiple large cystic follicles were found distended with 
follicular fluid in group II and squeezed the cortical stroma that affected the number of growing follicles in different stages of development, which was also found by the work of Ilyas et al. (2015). Further investigations of the ovarian stroma revealed the presence of large corpora lutea among the cystic follicles squeezing the stroma. The previous findings were in agreement with the findings of Robbins and Cotran (2014). The authors attributed the presence of large corpora lutea was due to a decrease in circulating thyroid hormones that are necessary for maintenance of differentiation of follicular granulosa cells to granulosa lutein cells.

In our study, it was noticed that the decreased numbers of growing follicles was accompanied by an increase in the numbers of atretic follicles that manifested the signs of apoptosis in agreement with the work of Regan et al. (2018) and proved the effect of altered thyroid hormones on the viability of the growing follicles.

Furthermore, marked condensation of interstitial cells among the cortical and subcortical stroma with luteinization of stromal cells was observed. In addition, the cytoplasm of stromal cells appeared vacuolated. These findings indicated that the thickening of the stromal cells (also known as hyperthecosis) was found to negatively influence folliculogenesis and provided further explanation for the decreased number of follicles in group II. These findings were also found by Robbins and Cotran (2014).

It should also be noted that in our present work, most of the rats of group II showed marked engorgement of both cortical and medullary blood vessels which was in accordance with $A b d-E l$ Fattah (2011) who attributed the engorgement of blood vessels to hyperactivity of the ovary in response to alterations in hormonal levels due to hypothyroidism.

Furthermore, the examination of the ovarian cortex of group III, showed a noticeable increase in the number of follicles of various stages of development accompanied by the presence of cystic follicles when compared to group II. In addition, congested medullary and cortical blood vessels were identified. These findings explained the reversible effect of cessation of Carbimazole intake and agreed with the results of Nida et al. (2016).

The immunohistochemical assessment of Vimentin for the control group showed that the granulosa cells were negatively stained in healthy primordial follicles, primary follicles and secondary (antral) follicles, with mild to patchy positive staining of large antral and cystic follicles. These findings disagree with the work of Maxie (2016) who stated that vimentin expression remained unchanged during follicular growth.

The granulosa cells of the cystic follicles were found to be more intense positively stained at the theca externa and near the periphery which decreased in pattern towards the center with positive staining of the follicular fluid. These findings agreed with the work of Serres et al. (2020) who stated that the dense staining of cystic follicles was probably linked to the structural and functional changes that occur during the process of cystogenesis. That confirmed the 
importance of vimentin for proper follicular growth and maturation.

It was further noticed that the granulosa cells within the corpus luteum of the control group showed moderate to intense positive staining besides the negative staining of the surrounding stroma along with intense positive staining around cortical and medullary blood vessels. These findings clarified the importance of vimentin in determining the viability of cells and agreed with Goel et al. (2018).

The atretic follicles showed weak brown staining and in some cases moderate focal staining that was distributed mainly at the periphery of the granulosa rather than towards the center. These findings agree with the work of Matsuda et al., (2012) who reported the same results and attributed them to the degeneration of the granulosa cell layer.

In addition, it was found that the stain was moderate to intense around some atretic follicles. The increase in intensity of the staining was supported by the findings of Singavarapu et al. (2010) that linked the changes in vimentin staining intensity to the degenerative change that occurred due to apoptosis and led to the shrinkage of the follicle.

The evaluation of the alpha smooth muscle actin's expression in the ovary of the control group revealed negative staining of the surface epithelium, tunica albuginea and interstitial cells with intense strong positive staining of the stroma surrounding the follicles and around blood vessels. Moreover, the follicles expressed various degrees of staining while the primordial, early and late primary was negatively stained. The granulosa of secondary follicles showed mild positive staining as well as moderate positive staining of the granulosa of antral and larger growing follicles as well as the follicular fluid. These findings agree with the work of Rodgers and Irving-Rodgers (2010) postulating that the presence of alpha smooth muscle actin is necessary for the contractile properties of the follicle that are necessary for increasing its size and may play a role in ovulation.

The rats of the experimental group II differed from the control group in faint staining around blood vessels and atretic follicles. In addition, the corpus luteum showed a more mild staining pattern than control group agreeing thus with the work of Arianmanesh et al. (2011) which considered the changes in representation of vimentin as a part of the cytoskeletal alterations leading to structural dysfunctions induced by hypothyroidism.

Furthermore, group II was found similar to the control group in the negative staining of the tunica albuginea, stroma, primordial, primary and secondary follicles. In addition, the growing follicles showed mild to negative staining of granulosa cells, which had the same distribution of the control group being more evident at the periphery more than at the center but appeared lesser in brown staining than the control group. This was confirmed by the work of DeSant'Ana et al. (2015).

The expression of alpha smooth muscle actin in the ovaries of group II showed negative staining of the surface epithelium, tunica albuginea, interstitial cells as well as the primary follicles with strong positive staining around blood vessels. The growing follicles were 
negatively stained and the corpora lutea showed focal to mild positive staining of the granulosa cells and intense staining of blood vessels within the corpora which agree with the work of Da Silva et al. (2018).

The corpus luteum in group III showed mild to patchy focal positive staining with negative staining of theca interna, theca externa, interstitial cells and the stroma surrounding the follicles. Moreover, the staining around atretic follicles was fainter in pattern than in group II. Besides, the staining around blood vessels was also denser in group II than in group III. In addition, negative staining of the surface epithelium, tunica albuginea were also noticed with strong positive staining of the blood vessels. The previous findings agreed with the findings of Hirschberg et al. (2012).

Furthermore, the follicles showed varying staining patterns, where the primordial follicles, early and late primary follicles and early secondary follicles were negatively stained. The granulosa of the late secondary follicles and antral follicles were positively stained with strong staining of the follicular fluid. The cystic follicle showed mild positive staining of the granulosa cells near the follicular cavity and negative staining near the periphery with intense positive staining of the theca externa, basal lamina and around blood vessels as well as around the atretic follicles which agreed with the results of De Sant'Ana et al. (2015).

Finally, the corpora lutea showed mild positive staining of the granulosa cells and intense staining of blood vessels within the corpora which agrees with the findings of Beindorff et al. (2010).

\section{CONCLUSION}

There was an observed change in the normal architecture of the ovary and various changes that occurred to the ovarian follicles including cystic formation and atresia that lead to a state of subfertility.

\section{Conflict of interest:}

The authors declare that they have no conflict of interest, commercial associations, or intent of financial gain regarding this research

\section{REFERENCES}

1. Abd-El Fattah (2011): Thyroid gland dysfunction modulates ovarian response to estrogen and androgen receptors in albino rats. The Egyptian Journal of Histology, 34:182190.

2. Ajayi A.F., Akhigbe R.E. and Ajayi L.O. (2013): Hypothalamic-pituitary-ovarian Axis in Thyroid Dysfunction, West Indian Med J, 62(9):835-838.

3. Arianmanesh M., McIntosh R.H., Lea R.G., Fowler P.A. and Al-Gubory K.H. (2011): Ovine corpus luteum proteins, with functions including oxidative stress and lipid metabolism, show complex alterations during implantation, Journal of Endocrinology, 210 (1): 47-58.

4. Beindorff N., Nagai K., Shirasuna K., Herzog K., Hoeffmann K.,Sasaki M., Bollwein H. and Miyamoto A. (2010): Vascular changes in the corpus luteum during early pregnancy in the cow, Journal of Reproduction \& Development 56:263-270.

5. Brent G.A. (2012): Mechanisms of thyroid hormone action. J Clin Invest., 122: 30353043.

6. Canipari R., Mangialardo C., Di Paolo V., Alfei F., Ucci S., Russi V., Santaguida M.G., Virili C., Segni M., Misiti S., Centanni M. and Falzacappa C.V. (2019): Thyroid 
hormones act as mitogenic and pro survival factors in rat ovarian follicles, Journal of Endocrinological Investigation, 42:271-282.

7. Da Silva A.C., Jammal M.P., Etchebehere R.M., Murta E.F.C. and Nomelini R.S. (2018): Role of alpha-smooth muscle actin and fibroblast activation protein alpha in ovarian neoplasms, Gnecol. Obstet. Invest., 83: 381-387.

8. De Sant'Ana F.J.F, Reis Junior J.L, Araújo R. L. S, Gimeno E. J. and Ortega H. H. (2015): Cytoskeletal proteins in the follicular wall of normal and cystic ovaries of sows, Pesq. Vet. Bras., 35(2):119-124.

9. Dittrich R., Matthias W. Beckmann M. W., Oppelt P.G., Hoffmann I., Lotz L., Kuwert T. and Mueller A. (2011): Thyroid hormone receptors and reproduction, Journal of Reproductive Immunology, 90 (1):58-66.

10. Gao J. (2020): P-values - a chronic conundrum, BMC Medical Research Methodology Journal, 167:1-8.

11. Goel A., Mohan Rao N., Santhi V., Sundar byna S., Grandhi B. and Conjeevaram J. (2018): Immunohistochemical Characterization of Normal Ovary and Common Epithelial Ovarian Neoplasm with a Monoclonal Antibody to Cytokeratin and Vimentin Iran J. Pathol., 13(1): 23-29.

12. Hapon M.B., Gamarra-Luques C. and Jahn G.A. (2010): Short term hypothyroidism affects ovarian function in the cycling rat, Reproductive Biology and Endocrinology, 8(14), 1-11.

13. Hirschberg R.M, Plendl J. and Kaessmeyer S. (2012): Alpha smooth muscle actin in the cycling ovary - an immunohistochemical study, Clinical Hemorheology and Microcirculation, 50(1-2):113-129.

14. Ilyas A., Ishaque I., Qamar N. and Parveen K. (2015): Effect of Experimentally Induced Hypothyroidism and its Treatment by Thyroxine on the Number of Follicles in an Ovary of Wistar Rats, Journal of Rawalpindi Medical College, 19(1):84-88.

15. Matsuda F., Inoue N., Manabe N. and Ohkura S. (2012): Follicular growth and atresia in mammalian ovaries: regulation by survival and death of granulosa cells. J. Reprod. Dev., 58:44-50.

16. Maxie G.M. (2016): Female Genital system in Jubb, Kennedy \& Palmer's Pathology of Domestic Animals, ElSevier, Chapter 4, 3(1): 358-464.

17. McDermott M.T. (2012): Does combination T4 and T3 therapy make sense? Endocr. Pract., 18 (5):750-757.

18. McKay G.A., Reid J.L. and Walters M.R. (2010): Clinical Pharmacology and therapeutics, 8th edition, Blackwell Publishing, Drugs and Endocrine diseases, 171-189.

19. Nelson, E.R., Allan, E.R., Pang, F.Y. and Habibi, H.R. (2011): Auto-regulation of thyroid hormone receptors in the goldfish ovary and testis. Gen. Comp. Endocrinol., 172(1): 50-55.

20. Nida Q. H., Shahnila N. and Mah Jabeen M. (2016): Original Article: The Effect of Hypothyroidism on the Body Weight of Adult Albino Wistar Rats, Journal of Rawalpindi Medical College, 20(2):147-149.

21. Patel M., Mishra V., Pawar V., Ramchandran R., Rajesh Sundar R. and Rajas Dabhi R. (2013): Evaluation of acute physiological and molecular alterations in surgically developed hypothyroid Wistar rats. J. Pharmacol. Pharmacother., 4(2):110-115.

22. Regan S.L.P., Knight P.G., Yovich J.L., Leung Y., Arfuso F. and Dharmarajan A. (2018): Granulosa Cell Apoptosis in the Ovarian Follicle: A Changing View. Front. Endocrinol., 9(61):1-10.

23. Robbins S.L. and Cotran R.S (2014): Pathologic basis of disease, Chapter 22: female genital tract; 10th edition, ElSevier, USA, 728-729.

24. Rodgers R.J. and Irving-Rodgers H.F. (2010): Morphological classification of bovine ovarian follicles, Reproduction, 139:309-318.

25. Salvetti N.R., Gimenoc E.J, Lorentea J.A. and Ortega H.H. (2004): Expression of Cytoskeletal Proteins in the Follicular Wall of 


\section{MOSTAFA M. MAHRAN et al.,}

Induced Ovarian Cysts, Cells Tissues Organs Journal, 178:117-125.

26. Serres M.P., Samwer M., Lavoie G., Perera U., Görlich D., Charras G., Roux P. and Paluch E.K. (2020): F-Actin Interactome Reveals Vimentin as a Key Regulator of Actin Organization and Cell Mechanics in Mitosis. Developmental Cell Journal, 52(2):210-222.

27. Silva J.F., Ocarino N.M. and Serakides R. (2018): Thyroid hormones and female reproduction, Biology of Reproduction Journal, 99(5):907-921.

28. Singavarapu R., Buchinsky N., Cheon D. and Orsulic S. (2010): Whole ovary immunohistochemistry for monitoring cell proliferation and ovulatory wound repair in the mouse, Reproductive Biology and Endocrinology, 8(98):1-7.

29. Treesh S.A and Khair N.S (2014): Effect of thyroid disorders on the adult female albino rats (Histological and Histochemical study), Journal of Histology and Cytology, 5(4):1-9.

30. Treuting P.M., Dintzis S.M. and Montine K.S (2018): Comparative anatomy and Histology, a mouse, rat and human atlas, 2nd Edition, El Sevier, USA, Chapter 17: Female reproductive system, 303-312.

31. Zaidi T.M., Khan A.A, Hasan B.M. and Faruqi A.N. (2004): Carbimazole Induced Thyroid Histopathy in Albino Rats during Development. J. Anat. Soc. India, 53 (2):1417. 
EFFECT OF CARBIMAZOLE ON THE STRUCTURE OF THE OVARY... 707

\section{تأثير عقار الكاربيمازول على المبيض في الجرذان البيضناء البالغة}

مصطفى محمد رفعت حسين مهران**، محب فريد منير رفلة*، حسن مصطقي سري*،

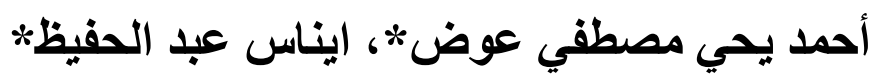

**قمم التشريح و علم الأجنة، كلية الطب البشري، جامعة مصر للعلوم و التكنولوجيا * قسم التشريح و علم الأجنة، كلية الطب البشري، جامعة عين شمس

E-mail: mostafamhtc@gmail.com

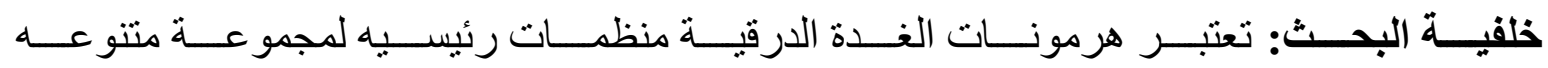

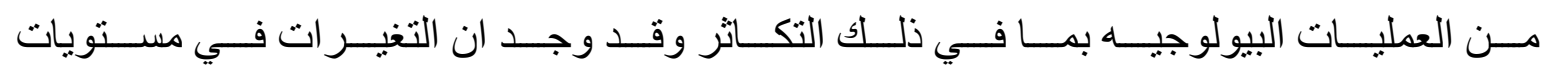

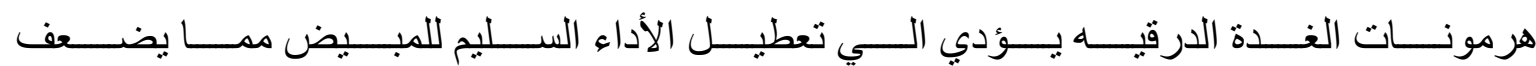

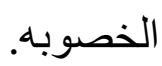

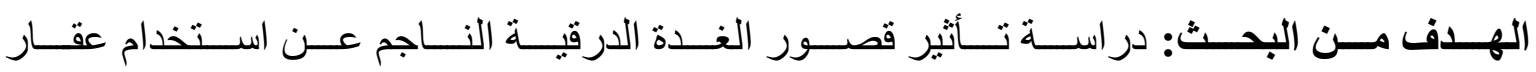

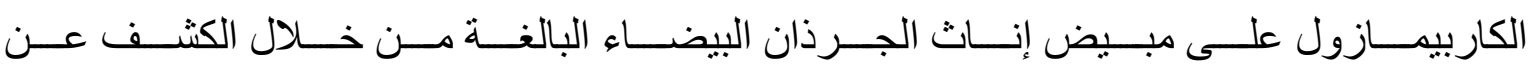
التغير ات الهيكلية بعد تناول عقار ألكاربيمازول وبعد التوقف عن تناوله.

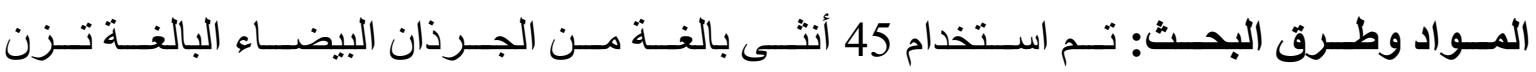

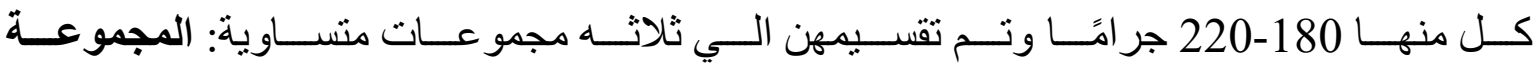

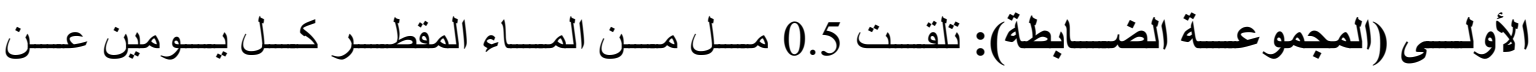

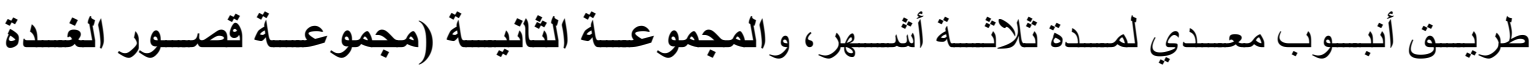

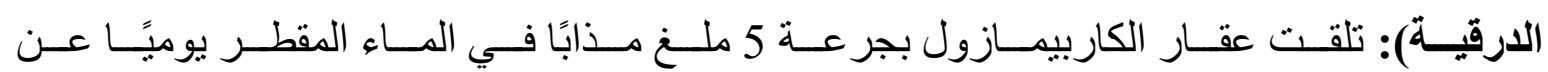

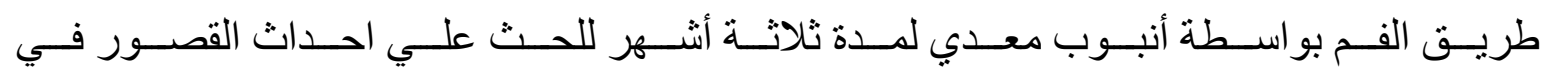

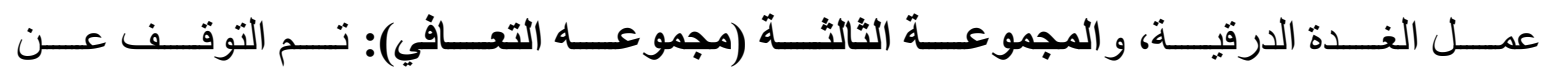

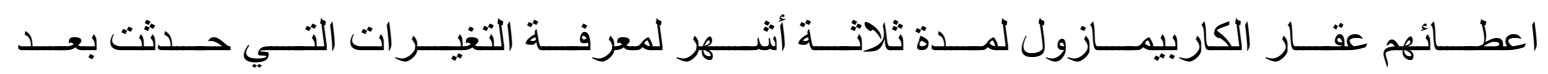

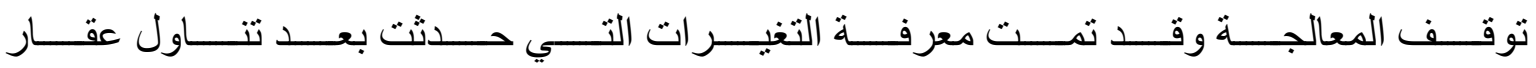

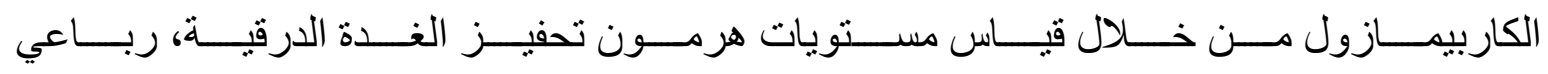

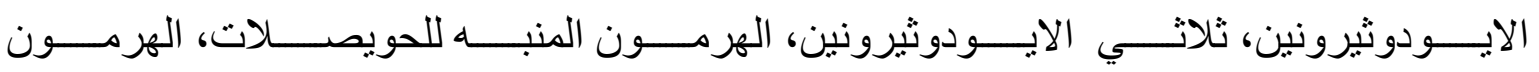

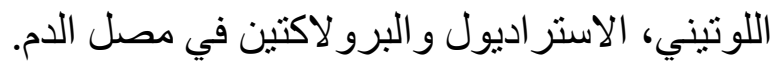




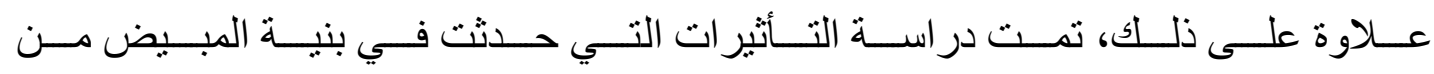

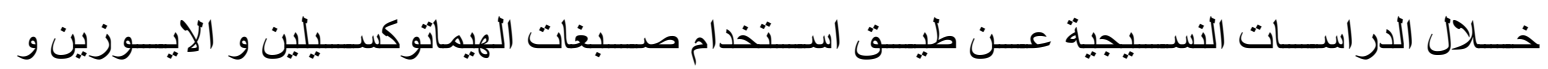

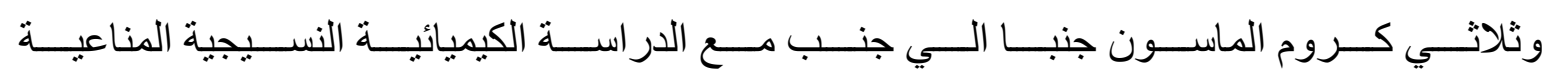
باستخدام الفيمنتين و الفا اكتين العضلات الملساء.

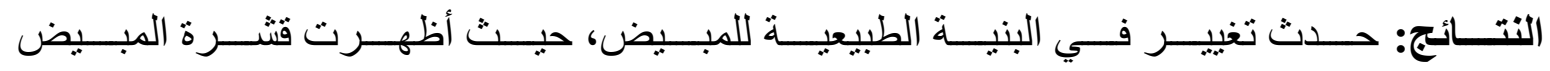

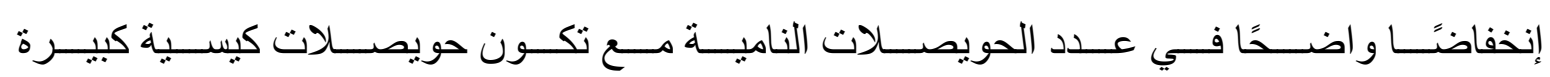

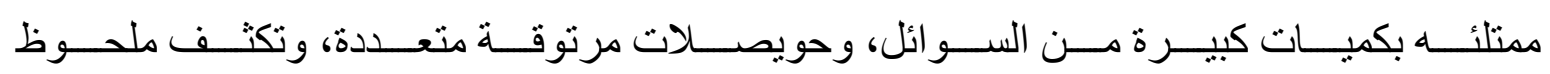

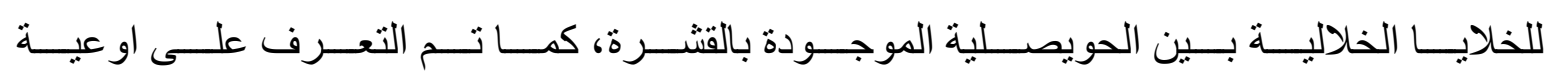

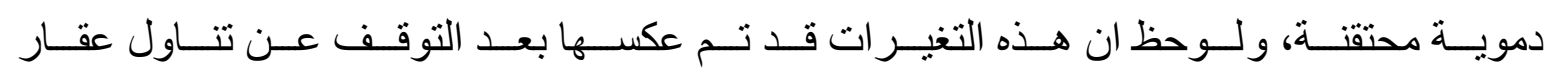

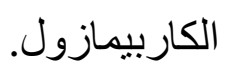

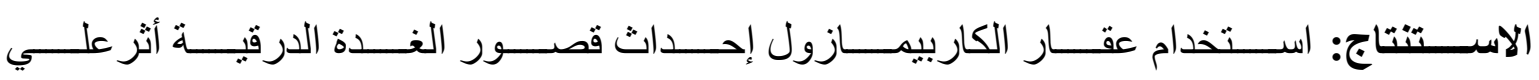
الوظيفة الطبيعية للمبيض من خلال العمل على مستويات مختلفة.

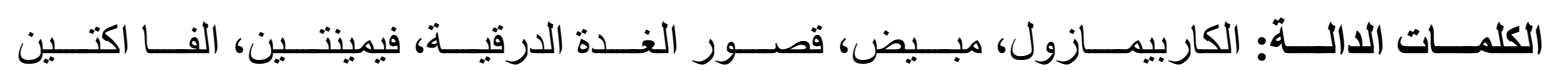
العضلات الملساء. 